\title{
Scientist Exchange Program
}

National Cancer Institute

\section{Source}

National Cancer Institute. Scientist Exchange Program. NCI Thesaurus. Code C16010.

Office of International Activities sponsors two international scientist exchange programs: Short-Term Scientist Exchange Program and Oncology Research Faculty Development Program 